\title{
A COMPTON-SUPPRESSION SPECTROMETER FOR $\gamma-\gamma$ COINCIDENCE MEASUREMENTS: LARGE SOLID ANGLE AND EXCELLENT SUPPRESSION
}

\author{
H.J.M. AARTS, C.J VAN DER POEL, D.E.C. SCHERPENZEEL, H.F.R. ARCISZEWSKI and G.A.P. \\ ENGELBERTINK
}

Fysisch Laboratorium, Ryksuniversitert Utrecht, PO Box 80.000, Utrecht, The Netherlands

Received 2 June 1980

For $\gamma-\gamma$ coincidence measurements a Compton-suppression spectrometer with a large solid angle of 120 msr and excellent suppression has been designed. The dimensions of the $\mathrm{NaI}$ anticoincidence shield have been optimized by means of Monte Carlo calculations. The NaI shield has a length of $35 \mathrm{~cm}$ and a diameter of $30 \mathrm{~cm}$. A $20 \%$ efficient HPGe crystal with a dead layer of $0.22 \mathrm{~mm}$ and a hollow core is used as central detector For ${ }^{60} \mathrm{Co}$, the average suppression of the Compton background between 100 and $1100 \mathrm{keV}$ amounts to 11.8 with a photopeak loss of $58 \%$. The areas of the 1173 and $1332 \mathrm{keV}$ peaks taken together amount to $60 \%$ of the total number of registered counts for the suppressed spectrum and to $15 \%$ for the non-suppressed spectrum. The spectrometer has been tested in a $\gamma-\gamma$ coincidence experiment with the reaction ${ }^{24} \mathrm{Mg}+(45 \mathrm{MeV}){ }^{16} \mathrm{O}$.

\section{Introduction}

In $\gamma$-ray spectroscopy with reactions induced by $\alpha$-particles or heavy ions, the complexity of the spectra and the low yield of many transitions of interest, call for spectrometers with high resolution, low background and large efficiency. The background due to Compton scattering can be removed to a great extend by surrounding the detector by an anticoincidence shreld.

Two different configurations of the shield have been reported [1-5]. The symmetrical Compton-suppression spectrometer [4] consists of a coaxial shield with a hollow core of the central detector. The incoming $\gamma$-ray beam is onented along the cylinder axis. The advantage of this geometry is the variable

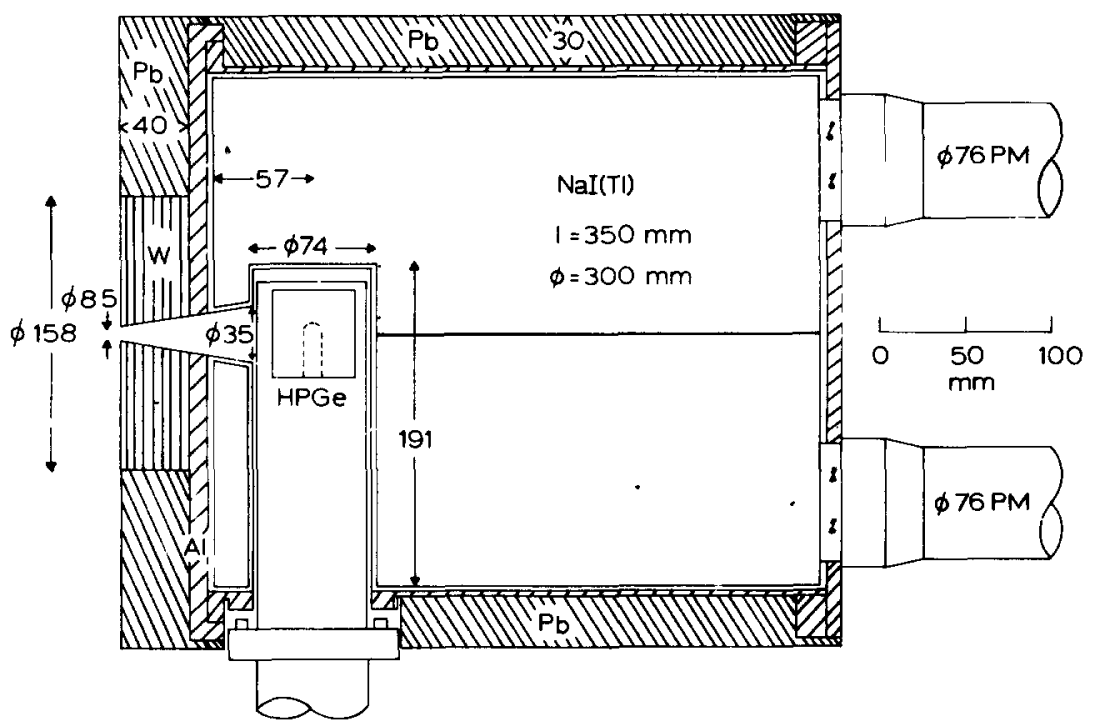

Fig. 1. Schematic drawing of the Compton suppression spectrometer with a solid angle of 120 ms The distance from the source to the central detector is $100 \mathrm{~mm}$. The dimensions are given in $\mathrm{mm}$ 
solid angle of the system which leads to a vanable average Compton suppression. It 1s, however, difficult to eliminate events which correspond to small-angle scattering and which leave little energy in the central detector. The geometry is especially disadvantageous for high-energy $\gamma$-rays, since Compton scattering is then peaked in the forward direction. The symmetr1cal set-up therefore leads to low Compton suppression in the low-energy part of the spectrum. In add1tion double-escape peaks are not suppressed very well, due to the presence of collnnear holes in the shield.

The above mentioned disadvantages are circumvented in the asymmetrical configuration shown in fig. 1, where the central detector is oriented at a right-angle to the direction of the incoming radiation.

Optimization of the Compton suppression of such an apparatus with disregard of the solid angle leads automatically to'a small photon entrance hole and to a relatively large distance between an external source and the central detector with, as a consequence, a rather small solid angle. Examples are the spectrometers of refs. [2] and [3] with solid angles of 7 and about $4 \mathrm{msr}$, respectively. These spectrometers are very suitable for singles measurements, such as those of a singles spectrum or of an angular distribution, where a large $\gamma$-ray flux compensates the small solid angle.

In $\gamma-\gamma$ coincidence experiments, however, in which a CSS is combined typically with three or four gating $\mathrm{Ge}\left(\mathrm{L}_{1}\right)$ detectors of about $400 \mathrm{msr}$ solid angle each, the combination is very poorly matched.

The present paper describes an asymmetrical CSS with a solid angle of $120 \mathrm{msr}$ and an excellent Compton suppression. The dimensions of the $\mathrm{NaI}$ anticoincidence shield are optımized by means of Monte Carlo calculations, which are described in ref. [5].

\section{Design}

The large solid angle of the spectrometer is obtained by making the minimum distance between the external source and the central crystal relatively short and the diameter of the $\gamma$-ray entrance hole large. The short minimum distance of course restricts the thickness of the $\mathrm{NaI}$ and the amount of passive shielding at the front side. In the present set-up the diameter of the entrance hole is limited by the dimensions of the central detector.

With a large solid angle chosen in this way, Monte

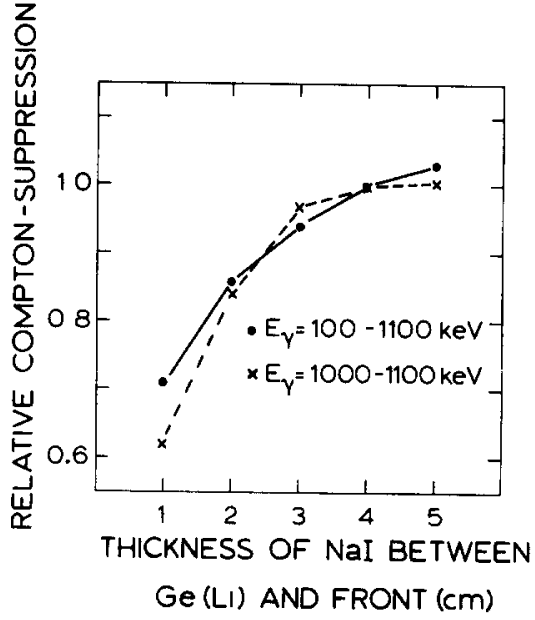

Fig. 2. The Monte Carlo result for the relative Compton suppression as a function of the thickness of $\mathrm{NaI}$ at the front side for ${ }^{60} \mathrm{Co}$ The energy regions $100-1100 \mathrm{keV}$ and $1000-$ $1100 \mathrm{keV}$ of the ${ }^{60} \mathrm{Co}$ spectrum of the central detector are considered. Both curves are arbitrarily normalized to unity for a thickness of $4 \mathrm{~cm}$.

Carlo calculations are performed to investigate the other dimensions of the asymmetrical CSS to obtain as good a Compton suppression as possible. The CSS configuration considered is shown in fig. 1 . The performance of this configuration has been studied as a function of the following parameters: the thickness of the $\mathrm{NaI}$ anticoincidence sheld at the front side,

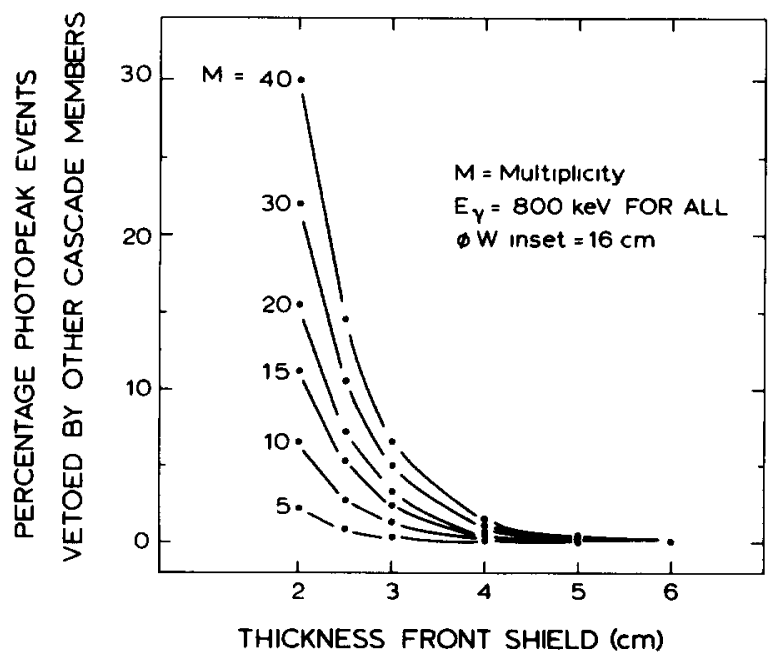

Fig. 3. The Monte Carlo result for cascade suppression as a function of the thickness of the passive front shield. The calculation has been performed for a cascade with multiplicity $M=5,10,15,20,30$ and 40 of which all members have an energy of $800 \mathrm{keV}$. The present front shield (see fig. 1) has a thickness of $4 \mathrm{~cm}$. 


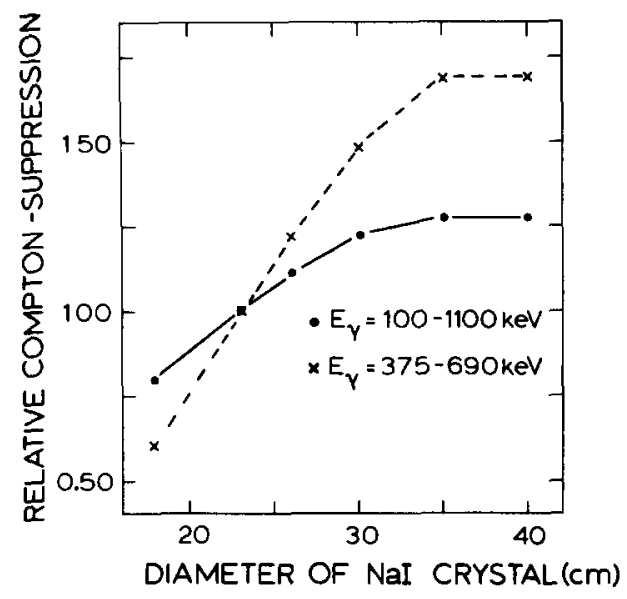

F1g. 4. The Monte Carlo result for the relative Compton suppression as a function of the diameter of the $\mathrm{NaI}$ sheld for ${ }^{60} \mathrm{Co}$. The energy regions $375-690 \mathrm{keV}$ and $100-1100 \mathrm{keV}$ of the ${ }^{60} \mathrm{Co}$ spectrum of the central detector are considered. Both curves are arbitrarily normalized to unity for a diameter of $23 \mathrm{~cm}$.

the amount of passive shielding, the diameter of the $\gamma$-ray entrance hole, the length and the diameter of the surrounding $\mathrm{NaI}$ anticoincidence shield.

The Monte Carlo calculations are similar to those reported in ref. [5]. The results are presented in figs. $2-5$ which show the relative Compton suppression as a function of the parameter considered. It should be remarked that the results are obtained with the other parameters kept fixed at the values given in fig. 1 .

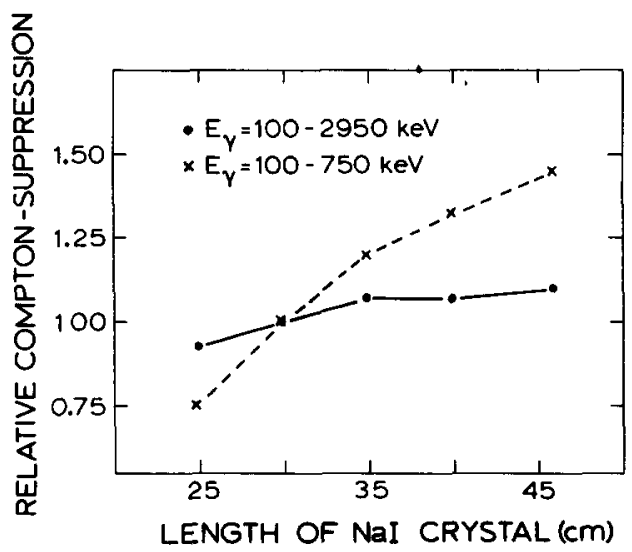

F1g. 5. The Monte Carlo result for the relative Compton suppression as a function of the length of the NaI shield for a $3 \mathrm{MeV} \gamma$-ray. The energy regions $100-750 \mathrm{keV}$ and $100-$ $2950 \mathrm{keV}$ of the spectrum of the central detector are considered Both curves are arbitrarly normalized to unity for a length of $30 \mathrm{~cm}$.

\subsection{The thickness of the NaI shield at the front side}

The main function of the NaI sheld between the central detector and the front of the spectrometer is to detect photons which are scattered over a large angle. Reduction of this part of the shield will therefore result in more Compton background close to the Compton edge. Fig. 2 gives the Monte Carlo result for the relative Compton suppression as a function of the thickness of $\mathrm{NaI}$ at the front side for ${ }^{60} \mathrm{Co}$. The energy regions $100-1100 \mathrm{keV}$ and $1000-1100 \mathrm{keV}$ of the ${ }^{60} \mathrm{Co}$ spectrum of the central detector are considered. Both curves are relative and arbitranly normalized to unity for a thickness of $4 \mathrm{~cm}$. In view of the short minimum distance desired, a thickness of $2 \mathrm{~cm} \mathrm{NaI}$ is chosen.

\subsection{The shielding lead front plate}

Reduction of the thickness of the lead front plate increases the probability for an unwanted photon to reach the anticoincidence sheld and to produce a veto signal. A $\gamma$-ray cascade of high multiplicity has to be considered in this respect. When a member of a cascade of multipolanty $M$ strikes the central detector then, angular correlation effects neglected, about $(M-1) / 2 \gamma$-rays will simultaneously hit the shielding front plate. Insufficient shelding at the front will thus cause suppression of $\gamma$-ray cascades with high multiplicity. The effective shielding of the front plate is increased by replacing the important central part of the lead by a $16 \mathrm{~cm}$ diameter inset of densimet, a W-Fe alloy $(1.2 \% \mathrm{Fe})$ with a density of $18 \mathrm{~g} / \mathrm{cm}^{3}$ (see fig. 1).

The Monte Carlo result for cascade suppression as a function of the thickness of the front plate is shown in fig. 3. The calculations have been performed for a cascade with multiplicity $M=5,10,15,20,30$ and 40 of which all members have an energy of $800 \mathrm{keV}$. Fig. 3 shows that for the chosen thickness of $4 \mathrm{~cm}$ the cascade suppression is less than $2 \%$, even for high multiplicitıes.

\subsection{The diameter of the $\gamma$-ray entrance hole}

Gamma-rays, which are scattered back from the central detector to the entrance hole, give rise to the inevitable Compton edge. A large-diameter entrance hole, a prerequisite for a large solid angle, leads necessarily to a relatively high Compton edge. Its position, 
however, is at a known energy distance from the corresponding photopeak.

In the present set-up the drameter of the entrance hole is chosen as large as allowed by the $50 \mathrm{~mm}$ diameter of a $20 \%$ efficient $\mathrm{Ge}$ detector. The entire central crystal is practically irradiated.

\subsection{The diameter and the length of the $\mathrm{NaI}$ anticoin- cidence shield}

Figure 4 shows (again for ${ }^{60} \mathrm{Co}$ ) the Monte Carlo result for the relative Compton suppression as a function of the diameter of the $\mathrm{NaI}$ shield. Apart from the overall suppression in the energy region 100-1100 $\mathrm{keV}$, the region from $375-690 \mathrm{keV}$ is also considered. In the latter the suppression is predominantly determined by the diameter of the shield as is shown in fig. 4. A diameter of $30 \mathrm{~cm}$ has been chosen.

The length of the anticoincidence shield mainly affects the suppression of photons which in the central crystal are scattered over a small angle. Since $\gamma$-rays of higher energy scatter more in the forward direction, a $\gamma$-ray of $3 \mathrm{MeV}$ is used to investigate the Compton suppression as a function of the length of the NaI crystal. Fig. 5 shows the result for the relative Compton suppression for the energy regions 100-
$750 \mathrm{keV}$ and $100-2950 \mathrm{keV}$. Both curves are arb1trarily normalized to unity for a length of $30 \mathrm{~cm}$. A length of $35 \mathrm{~cm}$ was chosen.

\subsection{The final configuration and electronics}

The geometry and dimensions of the $\mathrm{NaI}$ anticoinc1dence shield, discussed above, are shown in fig. 1 . The conical entrance hole corresponds to a position of the source at $16 \mathrm{~mm}$ in front of the spectrometer 1.e. the usual target position. The distance from the source to the central detector is now $10.0 \mathrm{~cm}$. The solid angle of the spectrometer amounts to $120 \mathrm{msr}$ or $0.96 \%$ of $4 \pi$. The half-angle subtended is $11^{\circ}$.

The NaI shield is divided into four optically separated sections, to allow a high count rate (up to $6 X$ $10^{5} \mathrm{~s}^{-1}$ ) in the shield without overloading the photomultıplier tubes. This division also makes it possible to use the system as a parr-spectrometer.

The anode signal of each of the four PM tubes is fed into a constant fraction discriminator (CFD, Canberra $1326 \mathrm{D}$ ) via a timing filter amplifier (TFA, ORTEC 454). The output pulses of the four CFDs are collected in a mixer which then produces the final veto signal (see fig. 6).

A modified CFD which generates the tıming signal

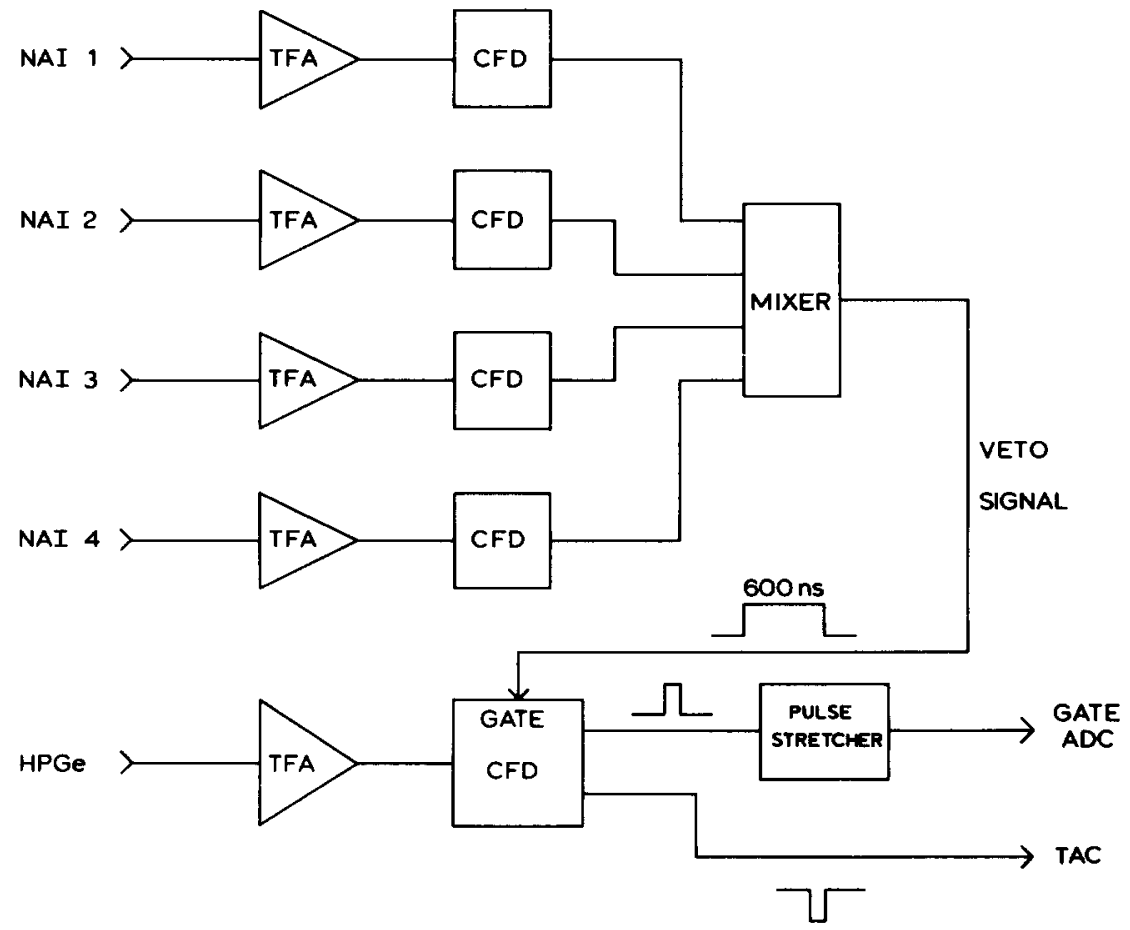

F1g 6. Block diagram of the electronics used, see section 2.5 


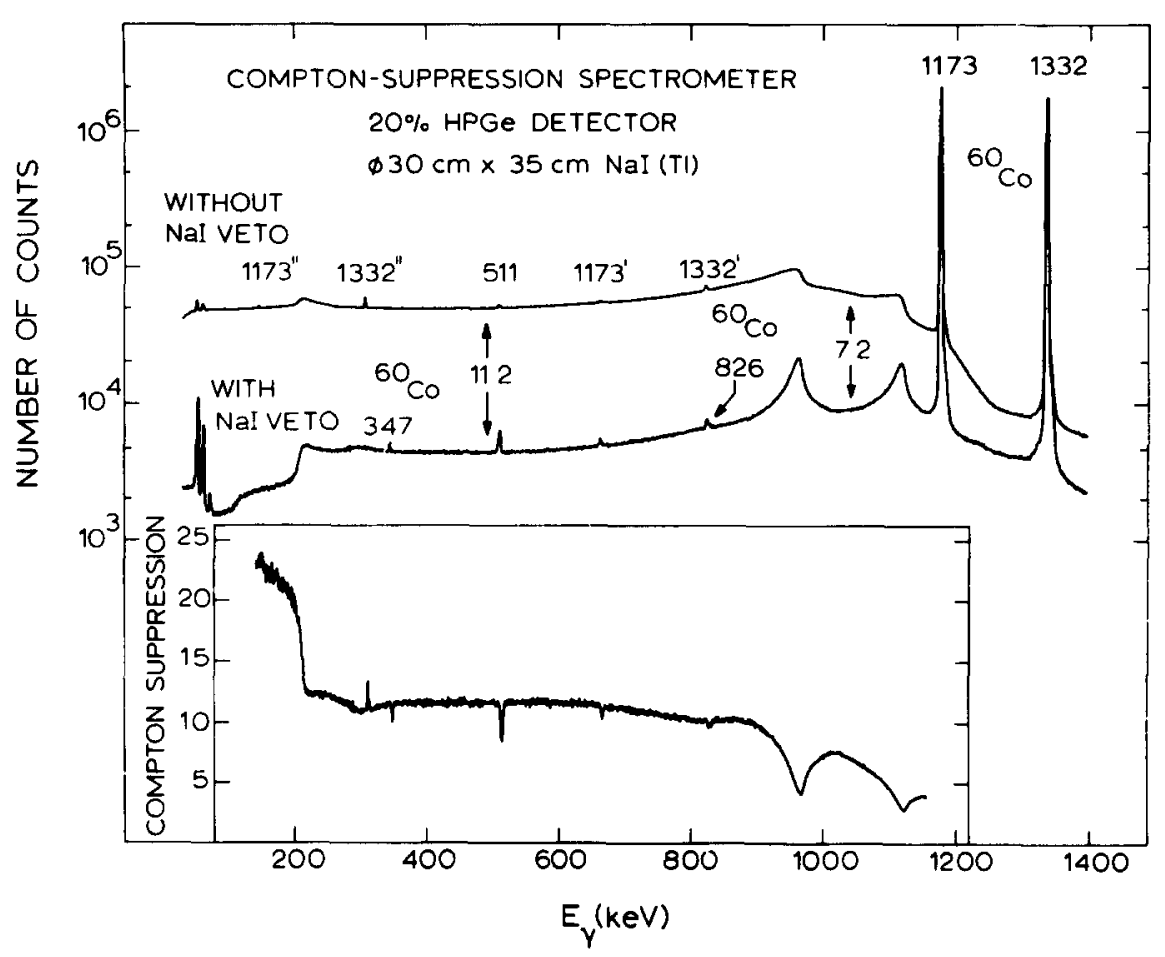

Fig. 7 Spectrum of ${ }^{60} \mathrm{Co}$, with and without Compton suppression. The inset gives the ratio between the channel contents of the upper and the lower spectrum, 1.e the Compton suppression as a function of the energy. The average Compton suppression between $100-1100 \mathrm{keV}$ amounts to 11.8 . The weak 347 and $826 \mathrm{keV}$ peaks belong to the ${ }^{60} \mathrm{Co}$ decay (see text). The relatively large Compton edges in the suppressed spectrum reflect the large solid angle.

of the central $\mathrm{Ge}$ detector is gated by the veto pulse, so that only non-vetoed timing signals are selected.

For a singles spectrum this selected timing signal 1s, after stretching, supplied to the gate input of an analog-to-digital converter (ADC) for prompt coinc1dence with the energy signal of the central detector.

In a coincidence experiment this selected timing signal is fed into the start of a time-to-amplitude converter (TAC).

\section{Performance of the spectrometer}

The pulse-height resolution of the four sections of the NaI sheld * varies between $11.8-12.9 \%$ for the $662 \mathrm{keV} \gamma$-ray from ${ }^{137} \mathrm{Cs}$. The lowest detectable energy above the noise is $28 \mathrm{keV}$. Coincidences between two sections give a time peak with a fwhm of $5.5 \mathrm{~ns}$.

A HPGe crystal * with a thin dead layer of $215 \mu \mathrm{m}$

* Manufactured by Harshaw Chemie B V, De Meern, The Netherlands. is used as a central detector. Such a crystal umproves the Compton suppression in the region of the Compton edge, as escape photons of low energy can easily reach the surrounding shield to produce a veto signal, see ref. [5]. The width of $600 \mathrm{~ns}$ for the veto signal is based on the time jitter of the HPGe signal and is chosen to guarantee a good overlap between the veto signal and the HPGe time pulse. This width therefore depends on the timing quality of the central detector.

\subsection{Results for ${ }^{60} \mathrm{Co}$ and ${ }^{56} \mathrm{Co}$}

To test the spectrometer spectra of ${ }^{60} \mathrm{Co}$ have been recorded with a $61 \mu \mathrm{C}_{1}$ source at the target pos1tion, $16 \mathrm{~mm}$ before the front plate (see fig. 1). Fig. 7 shows the spectra with and without Compton suppression. The spectra were accumulated for $1.5 \mathrm{~h}$. The non-suppressed count rate in the central detector was $24000 \mathrm{~s}^{-1}$, the suppressed count rate $6000 \mathrm{~s}^{-1}$. The large solid angle is reflected in the relatively large Compton edges in the suppressed spectrum. See for comparison fig. 5 of ref. [5] where the solid angle is a factor of 16 smaller. 


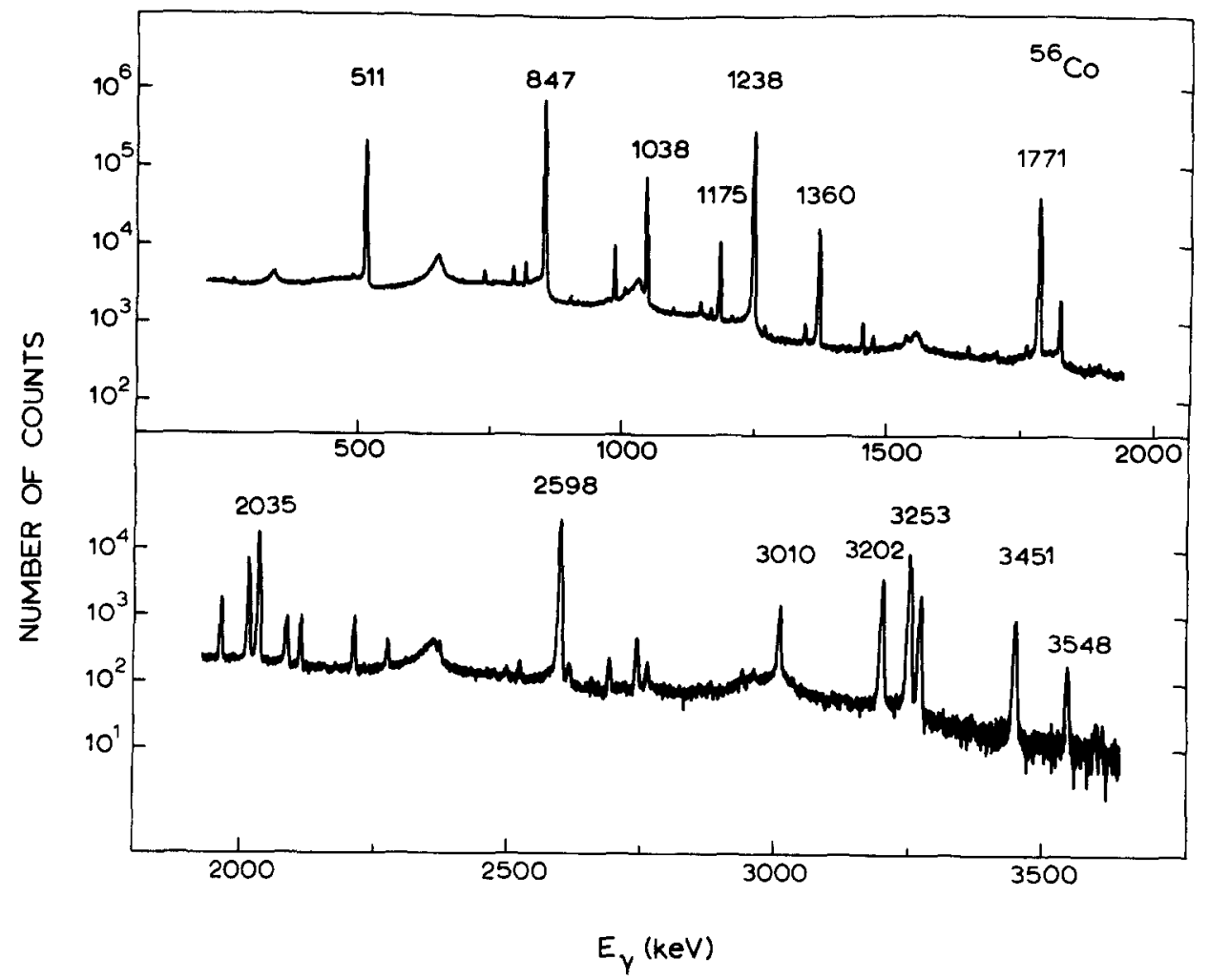

Fig. 8. Compton-suppressed spectrum of ${ }^{56} \mathrm{Co}$.

Figure 7 also gives the Compton suppression as a function of energy. The average Compton suppression between $100-1100 \mathrm{keV}$ amounts to 11.8 . The loss of pulses in the photopeaks amounts to $5.8 \%$.

The areas of the 1173 and $1332 \mathrm{keV}$ peaks taken together amount to $60 \%$ of the total number of

Table 1

Characteristic quantities of the Compton-suppression spectrometer with $120 \mathrm{msr}$ solid angle

Ant1-coincidence shield

Material

Dimensions

Lowest detectable energy

Central detector

Type

Dead layer thickness

Volume

Results for ${ }^{60} \mathrm{Co}$

Compton suppression for $100-1100 \mathrm{keV}$

Photopeak loss

Peak-to-total ratio
$\mathrm{NaI}$

See fig 1

$28 \mathrm{keV}$

HPGe

$215 \mu \mathrm{m}$

$90 \mathrm{~cm}^{3}$

118

$58 \%$

$60 \%$ registered counts for the suppressed spectrum and $15 \%$ for the non-suppressed spectrum.

The excellent Compton suppression is demonstrated by the appearance of the 347 and $826 \mathrm{keV}$ peaks, which belong to the ${ }^{60} \mathrm{Co}$ decay. The $347 \mathrm{keV}$ $\gamma$-ray originates from the same level as the $1173 \mathrm{keV}$ $\gamma$-ray, but with $I_{347} / I_{1173}=7.6 \times 10^{-5}$, see ref. [6]. The $826 \mathrm{keV} \gamma$-ray, a little higher in energy than the single-escape peak of $1332 \mathrm{keV}$, is the main branch from the ${ }^{60} \mathrm{~N}_{1}$ level at $2159 \mathrm{keV}$, which is fed by the $347 \mathrm{keV}$ transition.

Some characteristic quantities of the spectrometer are given in table 1.

Figure 8 shows the more complex spectrum of ${ }^{56} \mathrm{Co}$. The Compton edges associated with the strongest photopeaks are rather pronounced. This is the price to be paid for the large solid angle.

\subsection{In-beam $\gamma-\gamma$ coincidence results}

The spectrometer described here was designed for studies of discrete high-spin fast states of sd- and foshell nuclei. In these investigations, $\gamma-\gamma$ coincidence 


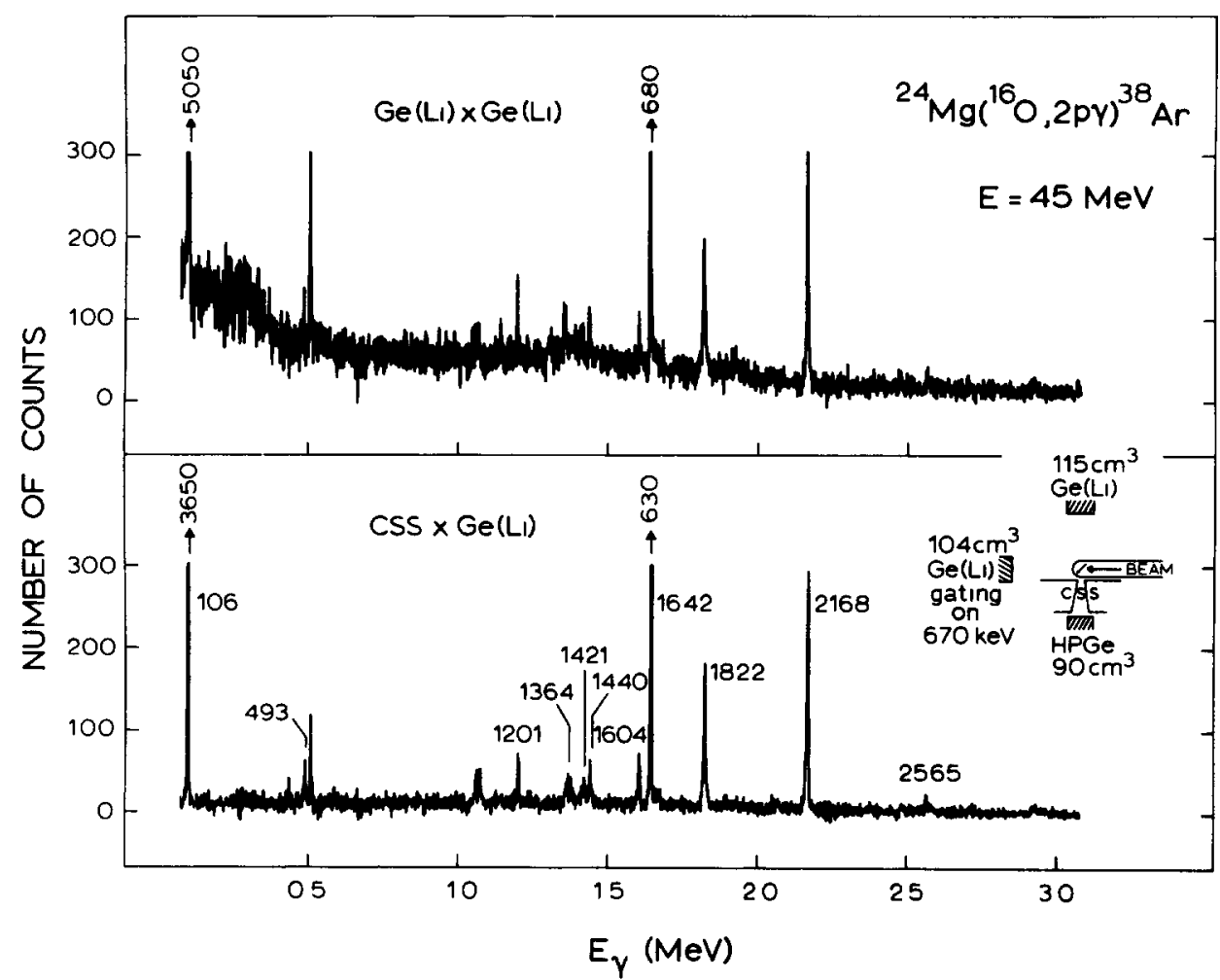

Fig 9. Spectra from the ${ }^{24} \mathrm{Mg}\left({ }^{16} \mathrm{O}, 2 \mathrm{p} \gamma\right){ }^{38} \mathrm{Ar}$ reaction, coincident with the $670 \mathrm{keV} \gamma$-ray observed at $0^{\circ}$ in the geometry as shown in the insert The upper coincidence spectrum 1 s recorded with a bare $115 \mathrm{~cm}^{3} \mathrm{Ge}\left(\mathrm{L}_{1}\right)$ detector at a distance of $11 \mathrm{~cm}$ The lower coincidence spectrum is recorded with the CSS The spectra are corrected for background and random phenomena (see section 3.2).

measurements play a major role [7-13]. To compare the present CSS with a bare $\mathrm{Ge}(\mathrm{Li})$ detector a $\boldsymbol{\gamma}-\boldsymbol{\gamma}$ coincidence experiment was performed. To faclitate the comparison, the set-up shown in the insert of fig. 9 was chosen. The CSS with $\mathrm{d} \Omega=120 \mathrm{msr}$ was placed at $\theta_{\gamma}=+90^{\circ}$ and a bare $\mathrm{Ge}\left(\mathrm{L}_{1}\right)$ detector with $\mathrm{d} \Omega=160 \mathrm{msr}(11 \mathrm{~cm}$ distance $)$ at $\theta_{\gamma}=-90^{\circ}$ The solid angles thus are about equal. A second bare $\mathrm{Ge}\left(\mathrm{L}_{1}\right)$ which serves as gate detector for the CSS as well as for the $115 \mathrm{~cm}^{3} \mathrm{Ge}(\mathrm{L})$ was situated at $\theta_{\gamma}=$ $0^{\circ}$ A $300 \mu \mathrm{g} / \mathrm{cm}^{2}{ }^{24} \mathrm{Mg}$ target, ennched to $99.94 \%$, on a $25 \mu \mathrm{m}$ Au backing was bombarded with a 45 $\mathrm{MeV}{ }^{16} \mathrm{O}^{6+}$ beam of $100 \mathrm{nA}$ (electrical). With the geometry, reaction, beam energy and target thickness given, the beam current was limited by the maximum count rate of about $25000 \mathrm{~s}^{-1}$ of the three Ge detectors. The real-to-random ratio in the time spectra $\mathrm{Ge}(\mathrm{Li}) \times \mathrm{Ge}(\mathrm{Li})$ and $\mathrm{CSS} \times \mathrm{Ge}(\mathrm{Li})$ was about 13 .

Durng the experiment a wide, approximate gate was set electronically around the $670 \mathrm{keV}$ peak in the gate detector at $\theta_{\gamma}=0^{\circ}$. The $670 \mathrm{keV} \gamma$-ray was pro- duced strongly in the decay of high-spin states in ${ }^{38} \mathbf{A r}$ (ref. [13]). The coincidence events, accumulated during $48 \mathrm{~h}$, are recorded on magnetic tape. In the offline analysis a proper (much narrower) gate is set on the $670 \mathrm{keV}$ peak. The final spectra, shown in fig. 9, have been corrected for background and random phenomena.

The data in fig. 9 clearly show the advantage of the present large-solid-angle CSS in $\gamma-\gamma$ coincidence measurements.

\section{Discussion}

The present spectrometer (fig. 1) combines a large solid angle of $120 \mathrm{msr}$ with an excellent Compton suppression. Other spectrometers reported recently in the literature have smaller solid angles and lower Compton suppression. A comparison is made in table 2.

The large solid angle makes the apparatus very 
Table 2

Comparison of some recent Compton-suppression spectrometers

\begin{tabular}{lll}
\hline Ref & $\begin{array}{l}\text { Solid angle } \\
(\mathrm{msr})\end{array}$ & $\begin{array}{l}\text { Average Compton } \\
\text { suppression } \\
\left({ }^{60} \mathrm{Co},\right. \\
100-1100 \mathrm{keV})\end{array}$ \\
& & $\approx 8$ \\
\hline Konijn et al [1] & $\approx 75$ & $10^{\mathrm{a}}$ \\
Van Driel et al [2] & 7 & $\approx 9$ \\
Beetz et al [3] & $\approx 4$ & $\approx 6$ \\
Lindblad [4] & 38 & 118 \\
Present spectrometer & 120 &
\end{tabular}

a See also ref [5] for use with a thin-dead-layer HPGe crystal as central detector.

suitable for $\boldsymbol{\gamma}-\boldsymbol{\gamma}$ coincidence measurements.

For the present spectrometer the $50 \mathrm{~mm}$ diameter of the central HPGe detector of $20 \%$ efficiency limits the diameter of the entrance hole of the $\mathrm{NaI}$ and thus the solid angle. A larger HPGe (or preferably a Gamma-X crystal, see ref. [5]) would allow an even bigger solid angle, also with'good Compton suppression. A further decrease of the thickness of the $\mathrm{NaI}$ at the front side, another possibility in order to increase the solid angle, is not recommended as it quickly leads to a deterioration of the Compton suppression as the Monte Carlo calculation in fig. 2 shows.

The maximum count rate for the present CSS under in-beam circumstances as described in section 3.2 is limited by the count rate which the central detector can handle. This in contrast with the apparatus described in refs. [2] and [5], where the count rate of the $\mathrm{NaI}$ anticoincidence shield was the limiting factor.

The average Compton suppression for $100-1100$ $\mathrm{keV}$ of ${ }^{60} \mathrm{Co}$ for the present CSS was calculated by Monte Carlo techniques to be 12.9 , a result reasonably close to the experimentally observed value of $11.8 \mathrm{in}$ view of the approximations in the calculation [5]. A loss of $0.8 \%$ of the veto pulses in the electronic circuitry (an effect not included in the Monte Carlo calculation) would also already explain the difference.

A characteristic quantity for a spectrometer in general with respect to Compton background is the peak-to-total ratio $P$, 1.e. the number of counts in the photopeaks divided by the total number of registered counts in the spectrum. The bare HPGe detector mentioned has for ${ }^{60} \mathrm{Co}$ a peak-to-total ratio of $P_{\mathrm{HP}}=$
0.15 . The present CSS has for ${ }^{60} \mathrm{Co}$ the value $P_{\mathrm{CSS}}=$ 0.60 Other quantities of interest can be expressed straightforwardly in $P_{\mathrm{HP}}$ and $P_{\mathrm{CSS}}$. For the central detector of the CSS, the ratio of non-suppressed count rate and suppressed count rate is e.g. given by $P_{\mathrm{CSS}} / P_{\mathrm{HP}}$.

In a $\gamma-\gamma$ coincidence experiment those events are of interest which correspond to a photopeakphotopeak coincidence. For two bare detectors with each $P=0.15$ the subset of photopeak-photopeak coincidences constitutes only $2.3 \%$ of the total number of coincidence events. For two Compton suppression spectrometers, however, each with $P_{\text {CSS }}=$ 0.60 , the photopeak-photopeak coincidences make up $36 \%$ of the total number of events. In this case the number of unwanted coincidence events written on magnetic tape is also reduced by the factor $\left(P_{\mathrm{CSS}} /\right.$ $\left.P_{\mathrm{HP}}\right)^{2}=16$.

The result in fig. 3 shows that the passive shielding at the front of the CSS is sufficiently thick to make cascade suppression negligible.

The performance of the spectrometer in a reaction which emits a large number of neutrons has not yet been investigated, but Lindblad [4] has successfully used a CSS in a study of the ${ }^{144} \mathrm{Nd}\left({ }^{12} \mathrm{C}, 6 n \gamma\right){ }^{150} \mathrm{Dy}$ reaction.

We like to thank D. Balke, J. Sodaar and N.A van Zwol for their technical contributions.

This work was performed as part of the research program of the "Stıchtıng voor Fundamenteel Onderzoek der Materie" (FOM) with financial support from the "Nederlandse Organisatie voor Zuiver Wetenschappelijk Onderzoek" (ZWO).

\section{References}

[1] J. Konijn, P.F A Goudsmit and E.W.A. Lingeman, Nucl. Instr. and Meth. 109 (1973) 83

[2] M A. van Driel, Thesis, R.U. Utrecht (1976).

[3] R. Beetz, W.L. Posthumus, F.W N. de Boer, J.L. Maarleveld, A. van der Schaaf and J. Konijn, Nucl. Instr. and Meth. 145 (1977) 353

[4] T. Lindblad, Nucl. Instr. and Meth 154 (1978) 53.

[5] H.J.M Aarts, G A.P Engelbertink, C.J. van der Poel, D.E.C. Scherpenzeel and H.F.R. Arciszewsk1, Nucl. Instr and Meth. 172 (1980) 439.

[6] D.C. Camp and J.R. van Hise, Phys. Rev C14 (1976) 261.

[7] M A. van Driel, G.A.P Engelbertınk, H.H. Eggenhuısen, L.P. Ekstrom and J A.J. Hermans, Phys Lett 59B (1975) 336 
[8] M.A. van Drıel, H.H Eggenhuisen, G.A.P. Engelbertınk, L.P. Ekstrom and J.A.J. Hermans, Nucl. Phys. A272 (1976) 466.

[9] L.P Ekstrom, H.H. Eggenhuisen, G A P Engelbertınk, J.A.J Hermans and H.J.M. Aarts, Nucl. Phys A283 (1977) 157.

[10] H H Eggenhuisen, L.P. Ekstrom, G.A P. Engelbertınk, H J.M. Aarts and J.A.J. Hermans, Nucl Phys A285 (1977) 167
[11] H.H. Eggenhuisen, L.P. Ekstrom, G.A.P. Engelbertınk, H.J.M. Aarts and W.G.J. Langeveld, Nucl. Phys. A299 (1978) 175.

[12] H.H Eggenhuisen, L.P. Ekstrom, G.A P. Engelbertınk and H.J.M. Aarts, Nucl. Phys. A 305 (1978) 245.

[13] H.J.M. Aarts, G.A.P. Engelbertink, H H. Eggenhuisen and L.P Ekstrom, Nucl. Phys A321 (1979) 515 